\title{
Utilization of the clinical laboratory for the implementation of concussion biomarkers in collegiate football and the necessity of personalized and predictive athlete specific reference intervals
}

Stefanie Schulte ${ }^{1,2}$, Natalie N. Rasmussen ${ }^{3,6}$, Joseph W. McBeth ${ }^{4}$, Patrick Q. Richards ${ }^{4}$, Eric Yochem ${ }^{4}$, David J. Petron ${ }^{5}$ and Frederick G. Strathmann ${ }^{3,6^{*}}$

\begin{abstract}
Background: A continued interest in concussion biomarkers makes the eventual implementation of identified biomarkers into routine concussion assessment an eventual reality. We sought to develop and test an interdisciplinary approach that could be used to integrate blood-based biomarkers into the established concussion management program for a collegiate football team.

Methods: We used a CLIA-certified laboratory for all testing and chose biomarkers where clinically validated testing was available as would be required for results used in clinical decision making. We summarized the existing methods and results for concussion assessment across an entire season to identify and demonstrate the challenges with the eventual integration of a parallel process using blood-based tests for concussion management. We analyzed the results of the biomarkers chosen for trends consistent with the outcome assessments provided from the current concussion management protocols.

Results: Baseline samples were collected with three additional post-concussion samples collected at three separate time points from players with a diagnosed concussion $(n=12)$. A summary of results from currently used concussion assessment tools were compared to the representative biomarkers S100B and NSE results. Nine sport-related concussions occurred during practice and three during play. For S100B, $50 \%$ had follow-up testing results lower than the post-injury result. In contrast, $92 \%$ of NSE follow-up results were lower than post-injury. One hundred percent of the results for S100B and NSE were within the athlete-derived reference intervals upon return-to-play and season end.

Conclusions: The reported workflow provides a framework for the eventual implementation of biomarkers for concussion assessment into existing assessment protocols and strengthens the need for reliance on clinical laboratory testing. Athlete-specific reference intervals will be required to adequately interpret results.
\end{abstract}

Keywords: Sports-related concussion, Biomarker panel, Predictive diagnostics, Blood test, Collegiate athletes, Football, Traumatic brain injury, S100B, NSE

\footnotetext{
* Correspondence: frederick.g.strathmann@aruplab.com

${ }^{3}$ Department of Pathology, University of Utah School of Medicine, 500

Chipeta Way mail code 115, Salt Lake City, UT 84108, USA

${ }^{6}$ ARUP Institute for Clinical and Experimental Pathology, ARUP Laboratories,

Salt Lake City, UT 84108, USA

Full list of author information is available at the end of the article
} 


\section{Background}

Sport-related concussion (SRC) continues to receive tremendous global attention. It has been estimated that up to 3.8 million SRCs occur in the USA each year [1]. Approximately $5.8 \%$ of all collegiate athletic injuries are SRCs with higher rates in contact sports such as football [2]. Recent findings suggest that collegiate football players who sustained one SRC in a season are more likely to sustain a second concussion in the same season compared to uninjured players. Additionally, collegiate football players are more likely to return-to play (RTP) early following concussion than athletes in other sports [3]. Accordingly, collegiate football players represent an important at risk population regarding concussion assessment and treatment.

Given the amount of SRC in collegiate football and the potential risk for long-term consequences associated with RTP, greater focus on the management and monitoring of SRC from multiple aspects is required and ongoing. Since various concussion laws endeavor to increase athletes' protection from the potential consequences of SRC, the Sports Concussion Management Policy of Utah High School Activities Association (UHSAA) serves as a standard for Utah's concussion management in high schools though collegiate athletics are arguably held to higher standards [4]. This policy recommends computerized neuropsychological assessment (CNA) which includes a pre-season baseline to allow comparison of concussed athletes' performance to their baseline values. Accordingly, the Concussion Program of the University of Utah, Department of Athletics implemented CNA as part of a comprehensive physical and neurocognitive assessment for Utah's collegiate football players [5]. Furthermore, the neurophysiological test Balance Error Scoring System (BESS) and the Sport Concussion Assessment Tool 2 ((SCAT2) at the time of manuscript preparation; currently superseded by SCAT3) are standardized sideline tools and follow-up evaluations for concussed football players. Additionally, a concussion symptom chart/checklist based on the University of Utah Sports Medicine Concussion Management Plan is used to monitor athletes' medical outcome. Even though these neurophysiological tests are relatively well-established testing methods in concussion management, recent studies have expressed concerns about the reliability and validity of neuropsychological tests for SRC assessment [6, 7]. Controversies regarding appropriate concussion diagnosis and management could potentially lead to premature RTP decisions [8]. Determining the RTP accurately can be challenging due the variability in presentation of concussion symptoms, and a lack of objective data indicating when full recovery has occurred $[9,10]$. Accordingly, the accurate diagnosis of SRC and determination of RTP criteria are crucial for a collegiate football player to recover from SRC.

The need for accurate diagnosis and management of $\mathrm{SRC}$ in an unbiased manner has resulted in efforts to identify blood-based biomarkers which are capable of documenting quantifiable biological changes directly related to physiological trauma $[11,12]$. The clinical use of blood-based biomarkers in addition to existing SRC assessment presents multiple advantages. Firstly, bloodbased biomarker measurement is minimally invasive and causes less cost for processing and analysis. Secondly, an accurate determination or exclusion of SRC would assist in the decision of whether further brain imaging or following-up testing is necessary. Thirdly, if appropriate biomarker concentrations could be correlated with the extent of SRC and progression of the injury, results could be used to objectively assess recovery time and RTP [13]. The majority of proposed biomarkers for concussion assessment have been identified and tested in the research setting. However, two extensively studied protein biomarkers for brain injury, S100B and neuron-specific enolase (NSE) [14], are available clinically. S100B concentrations of less than $0.1-0.12 \mu \mathrm{g} / \mathrm{l}$ have been shown to be a reliable tool to predict a normal CT scan and to support the clinician's decision not to perform CT imaging $[15,16]$. Furthermore, serum S100B concentrations greater than $0.027 \mathrm{ng} / \mathrm{mL}$ were found to significantly correlate with more than $90 \%$ of abnormal cerebrospinal-fluid albumin quotient results, the current gold standard for determining blood-brain barrier dysfunction (BBBD) [17]. Thus, the measurement of $\mathrm{S} 100 \mathrm{~B}$ is used in emergency room settings to rule out concussions $[15,18]$. Serum NSE levels of more than $16.32 \mu \mathrm{g} / \mathrm{L}$ are considered to be pathological [19]. NSE is a marker which directly reflects traumatic damage of the neurons [20]. Also, NSE has been shown to be $87 \%$ sensitive and $82.1 \%$ specific in predicting poor neurologic outcome in concussed patients [21]. In terms of predicting intracranial pathology and long lasting neurocognitive disability, early concentration peaks of both S100B and NSE have been shown to be sensitive indicators following concussion and BBBD [14, 22]. Thus, the markers S100B and NSE have demonstrated reasonable prognostic value for concussion management despite the existence of conflicting data [22, 23].

Given the potential value and promise of biomarker integration into athletic concussion assessment, the development of a successful workflow for their inevitable integration is crucial. The use of blood-based biomarkers in SRC management also implies the practicality of venipuncture and blood processing in situations and under circumstances that are not in line with the WHO guidelines [24]. Errors and delays in the blood collection and processing system can affect the diagnostic process [25]. In the present study, we sought to develop and test an interdisciplinary approach for the integration of blood-based biomarkers into the established concussion management program for the collegiate football team at the University of Utah using S100B and NSE as example 
biomarkers. The selection of S100B and NSE was based largely on their availability as clinically validated tests that could be conducted in a CLIA-certified central laboratory facility in contrast to a research setting. Herein, we present an established and successful interdisciplinary work flow for blood collection, on-site sample processing, and central laboratory testing for the duration of an entire collegiate football season. The included data provide a summary of the concussion assessment tools available at the time the study was conducted and demonstrate the successful integration of biomarker monitoring into collegiate athletics.

\section{Methods}

This project and its protocols were approved by the University of Utah Institutional Review Board (IRB \#00061977). The demographic summary for the included athletes (all male) was as follows: age range, 18 to 26 years; mean age, 21 years. For the baseline venipuncture, athletes were separated into two groups of approximately 60 athletes with the baseline collection occurring within $2 \mathrm{~h}$ post-physical activity that did not include full contact play. Five certified phlebotomists were present for the baseline venipuncture with each group of athletes taking approximately $1 \mathrm{~h}$ to complete. Individual venipuncture kits were organized to include all necessary materials for the venipuncture, and similar draw kits were used for the remaining venipunctures throughout the season (Additional file 1: Table S1). For post-injury venipunctures, two assistant athletic trainers with access to the athletes conducted phlebotomy training and met the required minimum for the observed venipunctures with certified phlebotomists prior to the start of the season. A Champion E-33 Series centrifuge (Ample Scientific LLC, Norcross, GA) was purchased for sample processing. Sample collection procedures were in line with those established for clinical testing including separation of the serum from the cells within $2 \mathrm{~h}$ and stored refrigerated until next day pick-up for transfer to the laboratory.

Samples were collected in red-top tubes, allowed to clot, and were separated from the cells within $2 \mathrm{~h}$ of being drawn. Aliquots were stored at $-70{ }^{\circ} \mathrm{C}$ until testing. $\mathrm{CanAg}^{\circ}$ NSE and S100B enzyme-linked immunosorbent assay kits (Fujirebio Diagnostics, Inc.,Göteborg, Sweden) were used following manufacturer directions provided in the package inserts, and testing was conducted in a CLIAcertified laboratory environment consistent with established laboratory Standard Operating Procedure. For the NSE test, 96-well plates were washed with a buffered wash solution, then $25 \mu \mathrm{L}$ of calibrators, QC material, and subject samples were added to each well in duplicate as per manufacturer recommendation. A $100 \mu \mathrm{L}$ of antibody solution was added to the wells and the plate was incubated, shaking for $1 \mathrm{~h}$ at room temperature. Following incubation, wells were rinsed with the buffered wash solution and $100 \mu \mathrm{L}$ of substrate was added. After $30 \mathrm{~min}$ of incubation at room temperature, $100 \mu \mathrm{L}$ of stop solution was added to each well. The plates were read at $405 \mathrm{~nm}$ using a Spectramax ${ }^{\circ}$ Plus384 spectrophotometer (Molecular Devices Corp., Sunnyvale, CA). A calibration curve of absorbance $(\mathrm{OD})$ versus concentration $(\mu \mathrm{g} / \mathrm{L})$ using a quadratic fit was generated by the plate reader software. Concentrations of QC and subject samples were automatically determined from the curve by the software. For the S100B test, 96-well plates were rinsed with a buffered wash solution. Fifty microliter of calibrators, QC material, and subject samples were added to each well in duplicate, followed by $100 \mu \mathrm{L}$ of biotin anti-S100. The plate was shaken at room temperature for $2 \mathrm{~h}$, and then rinsed with the wash buffer solution. A $100 \mu \mathrm{L}$ of tracer working solution was added to each well and the plate was incubated for $1 \mathrm{~h}$, shaking at room temperature. After incubating, wells were rinsed with buffered wash solution and $100 \mu \mathrm{L}$ of substrate was added. After $30 \mathrm{~min}, 100 \mu \mathrm{L}$ of stop solution was added and plates were read at $405 \mathrm{~nm}$ using the same spectrophotometer listed above. A calibration curve of absorbance (OD) versus concentration $(\mu \mathrm{g} / \mathrm{L})$ using a quadratic fit was generated by the plate reader software. Concentrations of $\mathrm{QC}$ and subject samples were automatically determined from the curve by the software. Analytical limits of quantification are $1-150 \mu \mathrm{g} / \mathrm{L}$ for NSE and $12-3500 \mathrm{ng} / \mathrm{L}$ for S100B.

Duplicate sample results all had CVs $<15 \%$; no repeats were necessary. Two levels of NSE QC material were pooled in house using de-identified patient samples previously tested for NSE. CanChek Tumor Marker Control Sera Levels 1 and 2 (IBL America, Inc.) were used as QC material for S100B. QC quantitated within two SDs of the mean established by the clinical laboratory for the current lots of material.

\section{Concussion assessment tools}

RTP decision management focuses mostly on signs and symptoms of neuropsychological test batteries [26, 27]. In accordance with Shane et al. [28], we chose to use the symptoms sections of the neuropsychological assessment tools. The symptom charts of SCAT2, CNA, and the University of Utah Sports Medicine Concussion Symptom Checklist compromise 22 similar symptoms with a symptom scoring from 0 (none) to 6 (severe).

\section{SCAT2}

The Sport Concussion Assessment Tool 2 (SCAT2) was developed to replace the original SCAT during the "3rd International Conference on Concussion in Sport" in 2008 and was in place at the time of manuscript preparation [29]. The SCAT2 was widely established as the 
standardized technique to evaluate, assess, and manage SRC for individuals older than 10 years with the end goal of safely returning the athlete to play [30]. SCAT2 enabled medical and health professionals to assess concussion in a standardized way by evaluating seven areas including symptoms, physical signs, Glasgow Coma Scale, sideline assessment using Maddocks score, cognitive assessment, balance, and coordination. The symptom chart of SCAT2 required the participant to score him/herself on 22 symptoms on a scale from 0 (none) to 6 (severe) based on how he or she is feeling. A total number of symptoms (max. 22) and a symptom severity score ( $\max .22 * 6=132$ ) is calculated. Furthermore, the participant was asked if physical or mental activity worsens the symptoms. In the event that the tester knows the participant well prior to the injury, he or she was asked how different the participant is acting compared to his or her usual self. The score of the symptom chart influences the total score of SCAT2. The SCAT3 is currently in use, and key differences are described elsewhere [30].

\section{Computerized neuropsychological assessment (CNA)}

Immediate Post-Concussion Assessment and Cognitive Testing (ImPACT) is the most widely used computerized neuropsychological test in the USA [31]. The ImPACT test compromises four sections including a demographic profile and health history questionnaire, current concussion symptoms and conditions, baseline and post-injury neurocognitive tests, and a graphic display of ImPACT test scores [32]. As in SCAT2, the symptom chart of ImPACT covers 22 symptoms with most of the symptoms being congruent and ranks the symptoms on a scale from 0 being "symptom free" to 6 being "severe".

\section{University of Utah Sports Medicine Concussion Symptom Checklist (CSC)}

In agreement with the SCAT2 and the CNA chart, the University of Utah Sports Medicine Concussion Symptom Checklist covers 22 symptoms. The symptoms and the scoring are identical with the CNA symptom chart. Each concussed student athlete completed the checklist with a certified athletic trainer daily. The total symptom score was used in assessing the athlete's progress and progression through the return-to-play protocol as defined in the University of Utah Sports Medicine Concussion Management Plan. When the symptom score was equal to zero, the player was no longer required to complete the symptom checklist. It was adopted from the CNA tool in an attempt to maintain continuity when comparing the symptom scores between the three different evaluation tools. This list and the CNA test could be completed over several different days, while the SCAT2 test was only administered at the time of a suspected SRC.

\section{BESS}

The Balance Error Scoring System (BESS) is a balance test developed to provide clinicians with an inexpensive and practical tool for the assessment of postural stability [33]. The BESS is frequently used for the assessment of postural stability in concussed athletes [34]. The test is comprised of three parts: double-leg stance (hands on the hips and feet together), single-leg stance (standing on the non-dominant leg with hands on hips), and a tandem stance (non-dominant foot behind the dominant foot) in a heel-to-toe fashion. Each of the three parts is executed on a firm and on a foam surface (eyes closed, $20 \mathrm{~s}$ each). The errors are counted during each trial, defined as (i) lifting hands off iliac crest, (ii) opening eyes, (iii) stepping, stumbling or falling, (iv) remaining out of

Table 1 Comparison of Symptom Charts used for concussion assessments

\begin{tabular}{|c|c|c|c|c|}
\hline & Symptoms & SCAT2 & CNA & $\operatorname{CsC}$ \\
\hline \multirow[t]{10}{*}{ General/cerebral } & Nausea or vomiting & * & $*$ & * \\
\hline & Dizziness & $*$ & * & * \\
\hline & Sensitivity to light & * & * & * \\
\hline & Sensitivity to noise & $*$ & * & * \\
\hline & Feeling slowed down & * & * & * \\
\hline & Feeling like "in a fog" & * & * & * \\
\hline & "Don't feel right" & * & & \\
\hline & Fatigue or low energy & * & * & $*$ \\
\hline & Confusion & * & & \\
\hline & Drowsiness & * & * & * \\
\hline \multirow[t]{4}{*}{ Pain/missensation } & Headache & * & * & * \\
\hline & "Pressure in head" & $*$ & & \\
\hline & Neck Pain & * & & \\
\hline & Numbness or tingling & & * & * \\
\hline \multirow[t]{5}{*}{ Impaired function } & Vision & $*$ & * & * \\
\hline & Balance & * & * & * \\
\hline & Concentration & * & * & * \\
\hline & Memory & * & * & * \\
\hline & Sleep & $* a$ & $* a, b$ & $* a, b$ \\
\hline \multirow[t]{4}{*}{ Emotions } & More emotional & * & * & * \\
\hline & Irritability & * & * & * \\
\hline & Sadness & * & * & * \\
\hline & Nervous or anxious & $*$ & * & * \\
\hline Other & & $* c, d$ & & $* c$ \\
\hline
\end{tabular}

The presented data compare the symptom charts of the Sport Concussion Assessment Tool 2 (SCAT2), the Computerized Neuropsychological Assessment (CNA), and the CSC (a symptom scale based on the University's Concussion Program). All symptom charts rank and score the symptoms on a scale from 0 (none) to 6 (severe)

${ }^{\text {a}}$ Trouble falling asleep

${ }^{b}$ Sleeping more/less than usual

'Symptoms get worse with physical/mental activity? (Y/N)

ds athlete acting different compared to the usual self? (no different/very different/unsure) 
Table 2 Time difference between the date of injury and post-injury assessments

\begin{tabular}{llll}
\hline & $\Delta$ DOI-DOA post & Mean \pm STDEV & Median \\
\hline BESS & $0-7 \mathrm{~d}$ & $1.8 \pm 2.2 \mathrm{~d}$ & $1.0 \mathrm{~d}$ \\
CNA & $1-7 \mathrm{~d}$ & $1.9 \pm 1.8 \mathrm{~d}$ & $1.0 \mathrm{~d}$ \\
SCAT2 & $15 \mathrm{~min}-158 \mathrm{hrs}$ & $28: 26 \pm 48: 48 \mathrm{hrs}$ & $5: 07 \mathrm{hrs}$ \\
CSC & $1-19 \mathrm{~d}$ & $4 \pm 5.5 \mathrm{~d}$ & $2.0 \mathrm{~d}$ \\
Biomarker (S100B and NSE) & 15 min-17:28 hrs & $5 \pm 7: 08 \mathrm{hrs}$ & $2: 00 \mathrm{hrs}$
\end{tabular}

This table displays the time difference $(\Delta)$ between the date of injury (DOI) and the date of the first concussion assessment following the injury (DOA post) using the most suitable unit of time in days (d), hours (hrs), and minutes ( $\mathrm{min}$ ). Mean and standard deviation are expressed in Mean \pm STDV. The assessment includes the Balance Error Scoring System (BESS), Computerized Neuropsychological Assessment (CNA), the Sport Concussion Assessment Tool 2 (SCAT2), the CSC (a symptom scale based on the University's Concussion Program) and the blood-based biomarkers S100 Beta (S100B) and neuron-specific enolase (NSE)

test position for more than $5 \mathrm{~s}$, (v) moving the hip into more than $30^{\circ}$ flexion or abduction, and (vi) lifting forefoot or heel [33].

\section{Statistics}

Given the nature of this study and the small sample size, the authors chose to analyze the data in a largely descriptive manner to avoid a misrepresentation and misinterpretation of the results [4]. ANOVA was performed for biomarker significance determination followed by a Tukey HSD post hoc analysis. Statistical analysis and figure generation were accomplished using $R$ [35] with the ggplot2 graphics package [36], respectively.

\section{Ethical considerations}

This project and its protocols were approved by the University of Utah Institutional Review Board (protocol \#00061977) and required informed consent for

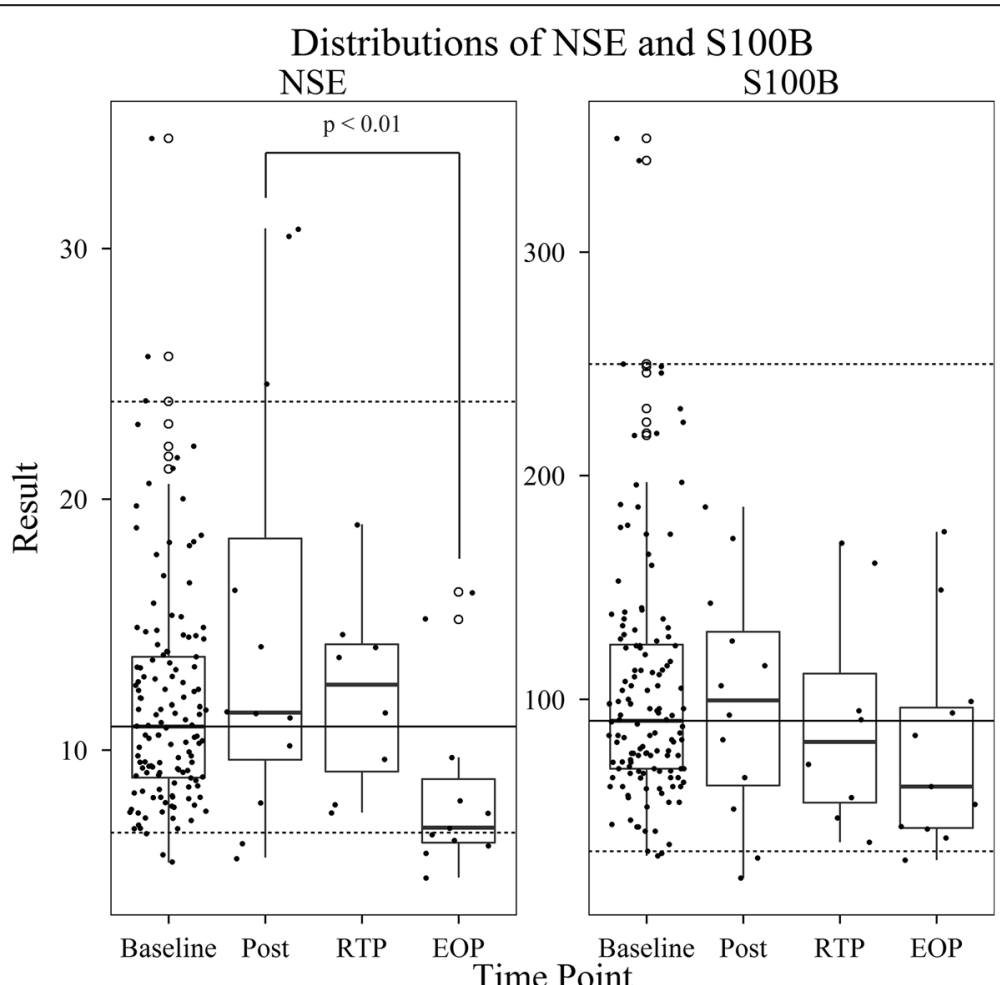

Fig. 1 NSE and S100B results from the baseline, post-injury (post), return-to-play (RTP) and end-of-play (EOP) venipunctures. Upper and lower hinges correspond to the 25th and 75th percentiles, respectively. The upper whisker extends from the 75th percentile to the highest value within 1.5 times the interquartile range (IQR). The lower whisker extends from the 25th percentile to the lowest value within 1.5 times the IQR. Data beyond the whiskers are outliers and plotted as open circles. Individual data points (solid black) are overlaid. The horizontal lower dashed line, solid black line, and upper dashed line represent the 2.5, 50, and 97.5 non-parametric percentiles, respectively, of the baseline results. The established reference interval that includes the middle $95 \%$ of the baseline data was determined to be 6.7 to $23.9 \mu \mathrm{g} / \mathrm{L}$ for NSE and 32 to $250 \mathrm{ng} / \mathrm{L}$ for S100B. A statistically significant difference was observed between the post and EOP venipunctures for NSE 
participation. All included athletes were consented at the time of the baseline sample blood draw event consistent with IRB requirements.

\section{Results}

A comparison of the Symptom Charts currently used is provided in Table 1 . In total, 12 SRCs in 11 athletes $(20.6 \pm 2.2$ years) were reported during the football season between August and November in 2013. Nine SRCs were reported occurring during a practice session and three during a play. Seven SRCs were caused by a direct hit to the athlete's head by another athlete's helmet (six) or a head collision with an object (one). Two SCRs were reported after a hard hit to the body, and three SRC reports were made after an unknown course of events (two) and after an unrelated injury (one). Less than half of the SRCs were self-reported (five). The majority was discovered by either an athletic trainer (five) or by the position coach (two). On average, concussed athletes began the return-to-play protocol after 2 days $(1.9 \pm 2.3$ days $)$ with a range of 3-48 days. For reasons of safety, two athletes were medically disqualified to return to play. RTP decisions were made based on a previously reported strategy consistent with current recommendations and guidelines [12].

As of the current state evaluation and a critical aspect to understanding how best to implement biomarkers into the workflow, we evaluated the time difference between the date of injury and the post-injury assessment for the concussion assessment tools in use. Summary data for the time differences for each Symptom Chart are provided in Table 2. In addition, a summary of the time difference between injury and the initial venipuncture are included. The time deference data for injury assessments and venipuncture indicate a wide distribution range that is consistent with the variability in symptom presentation and time to reporting of a sustained injury. The mean and median time differences for the post-injury venipuncture indicate adequate compliance with expectations of a venipuncture occurring within $2 \mathrm{~h}$ of the reported injury; however, the wide range further highlights the difficulty with reliance on self-reporting.

One of the challenges in applying clinical testing to a unique population such as collegiate athletes is the lack of suitable reference intervals for categorizing patient results in an appropriate context. Further, previously established "cutoffs" used in determining clinical sensitivity and specificity are expected to vary widely among differing populations and clinical circumstances. Figure 1 provides a summary of the NSE and S100B results for the baseline, post-injury, return-to-play and end-of-play venipunctures. In order to provide a population appropriate reference interval, baseline values $(n=127)$ were used to establish a non-parametric reference interval using the 2.5 and $97.5 \%$ of the data for NSE and S100B.
The established reference intervals were 6.7 to $23.9 \mu \mathrm{g} / \mathrm{L}$ for NSE and 32 to $250 \mathrm{ng} / \mathrm{L}$ for S100B. In comparison, reference intervals based upon healthy, non-athletes commonly used in clinical interpretations are 3.7 to $8.9 \mu \mathrm{g} / \mathrm{L}$ for NSE and 0 to $96 \mathrm{ng} / \mathrm{L}$ for S100B. A statistically significant difference was observed between the post-injury and end-of-play (EOP) results for NSE; however, a characteristic trend in the distribution of results for NSE and S100B was noted.

Although no clinical interpretation was applied to the NSE and S100B results during the season, we include the biomarker results in a summary table of outcome measures for athletes sustaining a diagnosed SRC in Table 3 and the individual results for each athlete in Table 4. Only the post-injury SCAT2 had $100 \%$ completion for all diagnosed SRCs among the concussion assessment tools while all venipunctures were completed at the required time points. Of the 12 diagnosed concussions, $100 \%(9 / 9)$ of the CNA and CSC assessments conducted at the RTP time point had lower symptom scores when compared to the post-concussive assessments consistent with approved protocols for RTP. For S100B, 50 \% (6/6) had RTP or EOS results lower than

Table 3 Combined outcome for concussion assessment tools and biomarker results for diagnosed SRCS

\begin{tabular}{lllllllll}
\hline Tool & Unit & POM & $n$ & Min & Max & Mean & STDEV & Median \\
\hline BESS & Error & Baseline & 11 & 4 & 25 & 12.2 & 5.0 & 11 \\
BESS & Error & Post & 11 & 5 & 37 & 16.7 & 9.3 & 13 \\
CNA & SC & Baseline & 12 & 0 & 15 & 3.3 & 5.6 & 0 \\
CNA & SC & Post & 11 & 0 & 89 & 25.0 & 28.3 & 18 \\
CNA & SC & RTP & 3 & 0 & 1 & 0.3 & 0.6 & 0 \\
SCAT2 & SC & Post & 12 & 2 & 102 & 39.1 & 28.6 & 34 \\
CSC & SC & Post & 9 & 0 & 91 & 22.1 & 31.4 & 7 \\
CSC & SC & RTP & 7 & 0 & 2 & 0.3 & 0.8 & 0 \\
S100B & ng/L & Baseline & 12 & 56 & 174 & 100.9 & 40.1 & 89 \\
S100B & ng/L & Post & 12 & 20 & 186 & 99 & 52.9 & 100 \\
S100B & ng/L & RTP & 12 & 28 & 170 & 78.6 & 45.8 & 66 \\
S100B & ng/L & EOS & 12 & 28 & 149 & 80.4 & 45.8 & 73 \\
NSE & $\mu \mathrm{g} / \mathrm{L}$ & Baseline & 12 & 5.8 & 21.7 & 13.5 & 5.1 & 14 \\
NSE & $\mu \mathrm{g} / \mathrm{L}$ & Post & 12 & 5.7 & 30.8 & 15.1 & 8.8 & 12 \\
NSE & $\mu \mathrm{g} / \mathrm{L}$ & RTP & 12 & 4.9 & 14.6 & 10.3 & 4.3 & 9 \\
NSE & $\mu \mathrm{g} / \mathrm{L}$ & EOS & 12 & 4.9 & 16.3 & 9.2 & 4.3 & 7 \\
\hline The & & & 12.3 & \\
\hline
\end{tabular}

The presented data provide an overview over the total outcome of the concussion assessment displayed by their unit (error during performance (Error), Symptom score (SC), nanogram per liter (ng/L), microgram per liter $(\mu \mathrm{g} / \mathrm{L})$, point of measurement (POM), sample size $(n)$, minimum (Min), maximum (Max), mean (Mean), and standard deviation (STDEV). The assessment includes the score from the Balance Error Scoring System (BESS), the score of the symptom charts of the Computerized Neuropsychological Assessment (CNA), the Sport Concussion Assessment Tool 2 (SCAT2), and the CSC (a symptom scale based on the University's Concussion Program), as well as the results of the blood-based biomarkers S100 Beta (S100B) and neuron-specific enolase (NSE) 
Table 4 Individual outcome Symptom Charts

\begin{tabular}{|c|c|c|c|c|c|c|c|c|c|c|c|c|c|c|}
\hline Tool & Unit & POM & 1 & 2 & 3 & 4 & 5 & 6 & 7 & 8 & 9 & 10 & $11^{a}$ & $12^{a}$ \\
\hline \multicolumn{15}{|c|}{ Concussion symptom assessment } \\
\hline BESS & Error & Baseline & 25 & 4 & 14 & * & 13 & 11 & 10 & 11 & 11 & 10 & 11 & 14 \\
\hline BESS & Error & Post & 30 & 11 & 20 & * & 37 & 14 & 13 & 13 & 11 & 18 & 5 & 12 \\
\hline CNA & SC & Baseline & 4 & 0 & 0 & 6 & 15 & 0 & 14 & 0 & 0 & 0 & 0 & 0 \\
\hline CNA & SC & Post & 30 & 0 & 40 & 1 & 18 & 58 & 10 & 3 & 0 & * & 26 & 89 \\
\hline CNA & SC & RTP & * & * & 0 & * & 0 & * & * & * & * & * & * & 1 \\
\hline SCAT & SC & Post & 46 & 9 & 102 & 23 & 27 & 66 & 2 & 30 & 12 & 50 & 38 & 64 \\
\hline $\operatorname{CSC}$ & SC & Post & 21 & 0 & * & 1 & 6 & 58 & * & 4 & 7 & 11 & * & 91 \\
\hline $\operatorname{CsC}$ & SC & RTP & 0 & * & * & 0 & 0 & 0 & * & * & * & 0 & 2 & 0 \\
\hline \multicolumn{15}{|c|}{ Biomarker } \\
\hline S100B & $\mathrm{ng} / \mathrm{L}$ & Baseline & 69 & 72 & 75 & 85 & 136 & 138 & 174 & 111 & 111 & 56 & 140 & 44 \\
\hline S100B & $\mathrm{ng} / \mathrm{L}$ & Post & 172 & 51 & 29 & 126 & 115 & 186 & 143 & 65 & 106 & 93 & 82 & 20 \\
\hline S100B & $\mathrm{ng} / \mathrm{L}$ & RTP & 47 & 36 & $\supset$ & 170 & 91 & 95 & 161 & 71 & 56 & $\supset$ & $\supset$ & $\supset$ \\
\hline S100B & $\mathrm{ng} / \mathrm{L}$ & EOS & 42 & 38 & 43 & 53 & 94 & 149 & 175 & 99 & 99 & 61 & 84 & 28 \\
\hline NSE & $\mathrm{ng} / \mathrm{mL}$ & Baseline & 12.3 & 17.8 & 18.3 & 10.1 & 18.9 & 11.4 & 7.8 & 14.9 & 14.9 & 5.8 & 21.7 & 7.6 \\
\hline NSE & $\mathrm{ng} / \mathrm{mL}$ & Post & 30.8 & 11.5 & 7.9 & 11.5 & 10.2 & 24.6 & 14.1 & 16.4 & 30.5 & 6.3 & 11.3 & 5.7 \\
\hline NSE & $\mathrm{ng} / \mathrm{mL}$ & RTP & 14.6 & 7.8 & $\supset$ & 9.6 & 7.5 & 14.1 & 11.5 & 13.7 & 19 & $\supset$ & כ & $\supset$ \\
\hline NSE & $\mathrm{ng} / \mathrm{mL}$ & EOS & 6.4 & 6.2 & 6.9 & 6.6 & 7.5 & 9.7 & 15.2 & 16.3 & 16.3 & 8 & 5.9 & 4.9 \\
\hline
\end{tabular}

The presented data show the individual outcome of the concussion assessment displayed by their unit (Error, Symptom score (SC), biomarker)), point of measurement (POM), sample size ( $n$ ), minimum (Min), maximum (Max), mean (Mean), and standard deviation (STDV). The assessment includes the score from the Balance Error Scoring System (BESS), the score of the symptom charts of the Computerized Neuropsychological Assessment (CNA), the Sport Concussion

Assessment Tool 2 (SCAT2), and the CSC (a symptom scale based on the University's Concussion Program), as well as the results of the blood-based biomarkers S100 Beta (S100B) and neuron-specific enolase (NSE). Missing values are displayed with the symbol "*". Some athletes were cleared to RTP at the EOS. Accordingly, the symbol " $\supset$ " indicates that the samples were drawn at the same time

${ }^{a}$ Athletes with labeled IDs (ID ${ }^{a}$ ) were medically disqualified to RTP

the post-injury result with the other $50 \%$ having higher results at the RTP or EOS time points when compared to the post-injury venipuncture. In contrast, $92 \%$ of NSE results (11/12) were lower at RTP or EOP time points when compared to the corresponding post-injury venipuncture. Of note, for athletes \#11 and \#12 who were medically disqualified and unable to return to play, the S100B concentration remained consistent with the post-injury result while NSE decreased in concentration. Interpretation of the data using the established reference intervals resulted in $100 \%$ of the baseline NSE and S100B values for the 12 injured athletes below the upper limit of the respective reference interval. For the postinjury samples, $100 \%$ of the S100B results were within the established reference interval while $25 \%(3 / 12)$ of the NSE results were above the upper limit of the reference interval. One hundred percent of the results for S100B and NSE were within the established reference intervals for the RTP and EOS samples.

\section{Conclusions}

In this study, we document the use of an interdisciplinary workflow for venipuncture, on-site sample processing, and central laboratory testing for the duration of an entire collegiate football season. Not surprisingly, in following a single, entirely male collegiate team, the overall sample size was limited in the number of concussions. In addition, our results provide an evaluation of concussion assessment tools in use at the time of manuscript preparation which did not include imaging studies as part of the treatment protocol. Although the overall utility of the biomarkers chosen for inclusion in our study remains controversial, we have developed a successful strategy in addressing the practical aspect of biomarker integration into the routine concussion assessment workflow. Further, by relying on clinically available assays, we provide an interdisciplinary approach with biomarker results generated under the requirements of laboratory testing with well-defined criteria for accuracy, reproducibility, and assay performance not required in the research setting [12, 37].

Although the primary objective of the current study was not to address the utility of S100B or NSE in diagnosing SRC, the results of the baseline venipuncture and the reference intervals established from the dataset indicate that for both S100B and NSE, the currently used reference intervals are likely not applicable to athletes. This finding is a common occurrence in laboratory medicine that often prompts the necessary establishment of population specific reference intervals. In the context of physical activity, interpretation of established reference intervals 
can be further complicated $[38,39]$ and necessitates tight control over experimental design and sample collection for research as well as a well-defined protocol for eventual implementation.

The introduction of biomarkers into athletics for SRC diagnosis is a multi-faceted process and success of any proposed biomarker or biomarker panels will rest largely on the capability of successful implementation into the current concussion assessment workflows. Although much attention has been focused on biomarker discovery, the presented study was designed to investigate the practicality of biomarker implementation in a routine concussion assessment workflow including venipunctures at key time points before and after injury in addition to clinical assessments made for RTP for an entire collegiate football team.

\section{Expert recommendations}

As the discovery of novel biomarkers and biomarker panels continues, successful implementation will rely on a thorough understanding of the constraints of the entire system and how best to enact required protocols. A continued need exists to identify and implement biomarkers with utility in all phases of the injury cycle with careful attention given to the practical limitations inherent to the nature of athletic competition.

\section{Additional file}

Additional file 1: Table S1. Itemized venipuncture kits. (DOCX $14.2 \mathrm{~kb}$ )

\section{Abbreviations}

BESS: Balance Error Scoring System; NSE: neuron-specific enolase; RPT: computerized neuropsychological assessment; RPT: return to play; SCAT2: Sport Concussion Assessment Tool 2; SRC: sports-related concussion.

\section{Competing interests}

The authors declare that they have no competing interests.

\section{Authors' contributions}

FS designed the collection workflow, oversaw clinical testing, coordinated the study, prepared the manuscript; SS helped design the collection workflow, prepared the manuscript, and conducted the data analyses; NR conducted all clinical tests and participated in the blood collection; JM, PR, and EY oversaw and/or participated in the blood collections and sample processing during the season; DP provided clinical oversight for the study. All authors participated in manuscript preparation and editing. All authors read and approved the final manuscript.

\section{Authors' information}

David Petron is the team physician for the University of Utah Athletics. Frederick Strathmann is a medical director at ARUP Laboratories and is board certified in clinical chemistry and toxicological chemistry from the American Board of Clinical Chemistry.

\section{Acknowledgements}

The authors would like to thank the Departments of Sports Medicine, Orthopaedics and Pathology at the University of Utah for financial support. FS would like to thank Dr. Bradley Hayes, the ARUP Institute for Clinical and Experimental Pathology, Dr. David Grenache, and the Special Chemistry Laboratory at ARUP Laboratories.

\section{Author details}

'Department of Psychology, University of Utah School of Medicine, Salt Lake City, UT 84108, USA. ${ }^{2}$ Department of Exercise and Sport Science, University of Utah School of Medicine, Salt Lake City, UT 84108, USA. ${ }^{3}$ Department of Pathology, University of Utah School of Medicine, 500 Chipeta Way mail code 115, Salt Lake City, UT 84108, USA. ${ }^{4}$ Department of Athletics, University of Utah School of Medicine, Salt Lake City, UT 84108, USA. ${ }^{5}$ Department of Orthopaedics, University of Utah School of Medicine, Salt Lake City, UT 84108, USA. ${ }^{6}$ ARUP Institute for Clinical and Experimental Pathology, ARUP Laboratories, Salt Lake City, UT 84108, USA.

Received: 27 November 2015 Accepted: 12 January 2016

Published online: 27 January 2016

\section{References}

1. Halstead ME, Walter KD. American Academy of Pediatrics. Clinical report-sport-related concussion in children and adolescents. Pediatrics. 2010;126(3):597-615. doi:10.1542/peds.2010-2005.

2. Gessel LM, Fields SK, Collins CL, Dick RW, Comstock RD. Concussions among United States high school and collegiate athletes. J Athl Train. 2007;42(4):495-503.

3. Iverson GL, Gaetz M, Lovell MR, Collins MW. Cumulative effects of concussion in amateur athletes. Brain Inj. 2004;18(5):433-43. doi:10.1080/02699050310001617352.

4. Arain M, Campbell MJ, Cooper CL, Lancaster GA. What is a pilot or feasibility study? A review of current practice and editorial policy. BMC Med Res Methodol. 2010:10:67. doi:10.1186/1471-2288-10-67.

5. UTE Conference I. Concussion Management Plan. 2014. http://www.utefootball. org/ute2011/webdocs2011/uteconcussionstatement.pdf. Accessed March 232014.

6. McCrea M. An independent, prospective, head to head study of the reliability and validity of neurocognitive test batteries for the assessment of mild traumatic brain injury: Medical Coll of Wisconsin Milwaukee 2013.

7. Szabo AJ, Alosco ML, Fedor A, Gunstad J. Invalid performance and the ImPACT in national collegiate athletic association division I football players. J Athl Train. 2013:48(6):851-5. doi:10.4085/1062-6050-48.6.20.

8. Crutchfield KE, Ferrell JL. Controversies in concussion management: who should clear the athlete to return to play? Md Med. 2011:12(1):13-4. 20.

9. McKeon JM, Livingston SC, Reed A, Hosey RG, Black WS, Bush HM. Trends in concussion return-to-play timelines among high school athletes from 2007 through 2009. J Athl Train. 2013;48(6):836-43. doi:10.4085/1062-6050-48.6.17.

10. Neselius S, Brisby H. Sport-related concussions: time to diversify care and recommendation advice. Knee Surg Sports Traumatol Arthrosc. 2014;22(2): 233-4. doi:10.1007/s00167-013-2792-5.

11. Marchi N, Bazarian JJ, Puvenna V, Janigro M, Ghosh C, Zhong J, et al. Consequences of repeated blood-brain barrier disruption in football players. PLoS One. 2013;8(3):e56805. doi:10.1371/journal.pone.0056805.

12. Strathmann FG, Schulte S, Goerl K, Petron DJ. Blood-based biomarkers for traumatic brain injury: evaluation of research approaches, available methods and potential utility from the clinician and clinical laboratory perspectives. Clin Biochem. 2014;47(10-11):876-88. doi:10.1016/j.clinbiochem.2014.01.028.

13. Jeter CB, Hergenroeder GW, Hylin MJ, Redell JB, Moore AN, Dash PK. Biomarkers for the diagnosis and prognosis of mild traumatic brain injury/ concussion. J Neurotrauma. 2013;30(8):657-70. doi:10.1089/neu.2012.2439.

14. Pandor A, Goodacre S, Harnan S, Holmes M, Pickering A, Fitzgerald P, et al. Diagnostic management strategies for adults and children with minor head injury: a systematic review and an economic evaluation. Health Technol Assess. 2011;15(27):1-202.

15. Zongo D, Ribereau-Gayon R, Masson F, Laborey M, Contrand B, Salmi LR, et al. S100-B protein as a screening tool for the early assessment of minor head injury. Ann Emerg Med. 2012;59(3):209-18. doi:10.1016/j.annemergmed.2011.07.027.

16. Unden J, Romner B. Can low serum levels of S100B predict normal CT findings after minor head injury in adults? An evidence-based review and meta-analysis. J Head Trauma Rehabil. 2010;25(4):228-40.

17. Blyth BJ, Farhavar A, Gee C, Hawthorn B, He H, Nayak A, et al. Validation of serum markers for blood-brain barrier disruption in traumatic brain injury. J Neurotrauma. 2009;26(9):1497-507. doi:10.1089/neu.2008-0738.

18. Taira BR, Schriger DL. Annals of Emergency Medicine Journal Club. Biomarkers revisited: study design, validity, and STARD: Will S100-B affect computed tomography use in head injury patients?: Answers to the March 2012 Journal Club questions. Ann Emerg Med. 2012;60(2):236-40. doi:10.1016/j.annemergmed.2012.04.004. 
19. Wolf H, Frantal S, Pajenda GS, Salameh O, Widhalm H, Hajdu S, et al. Predictive value of neuromarkers supported by a set of clinical criteria in patients with mild traumatic brain injury: S100B protein and neuron-specific enolase on trial: clinical article. Journal Neurosurg. 2013;118(6):1298-303. doi:10. 3171/2013.1.JNS121181.

20. Stalnacke BM, Tegner $Y$, Sojka P. Playing soccer increases serum concentrations of the biochemical markers of brain damage S-100B and neuron-specific enolase in elite players: a pilot study. Brain Inj. 2004;18(9):899-909. doi:10.1080/02699050410001671865.

21. Meric E, Gunduz A, Turedi S, Cakir E, Yandi M. The prognostic value of neuron-specific enolase in head trauma patients. The J Emerg Med. 2010; 38(3):297-301. http://dx.doi.org/10.1016/j.jemermed.2007.11.032.

22. Topolovec-Vranic J, Pollmann-Mudryj MA, Ouchterlony D, Klein D, Spence J, Romaschin A, et al. The value of serum biomarkers in prediction models of outcome after mild traumatic brain injury. J Trauma. 2011;71(5 Suppl 1):S478-86. doi:10.1097/TA.0b013e318232fa70.

23. Shahim $P$, Tegner $Y$, Wilson DH, Randall J, Skillback T, Pazooki D, et al. Blood biomarkers for brain injury in concussed professional ice hockey players. JAMA Neurol. 2014;71(6):684-92. doi:10.1001/jamaneurol.2014.367.

24. World Health Organization. WHO guidelines on drawing blood: best practices in phlebotomy. Geneva. 2010. http://www.ncbi.nlm.nih.gov/pubmed/ 23741774. Accessed March 23.

25. Mendonca B, Phibbs A, Vandermeer J, Pasek ZJ. Use of simulation in support of analysis and improvement of blood collection process. Proceedings of the Winter Simulation Conference. Baltimore, Maryland: 2433798: Winter Simulation Conference; 2010. p. 2352-63.

26. McCrory P, Meeuwisse WH, Aubry M, Cantu B, Dvorak J, Echemendia RJ, et al. Consensus statement on concussion in sport: the 4th International Conference on Concussion in Sport held in Zurich, November 2012. Br J Sports Med. 2013:47(5):250-8. doi:10.1136/bjsports-2013-092313.

27. Darling SR, Leddy JJ, Baker JG, Williams AJ, Surace A, Miecznikowski JC, et al. Evaluation of the Zurich guidelines and exercise testing for return to play in adolescents following concussion. Clin J Sport Med. 2014;24(2):128-33. doi: 10.1097/JSM.0000000000000026.

28. Shane ER, Pierce KM, Gonzalez JK, Campbell NJ. Sports chiropractic management of concussions using the Sport Concussion Assessment Tool 2 symptom scoring, serial examinations, and graded return to play protocol: a retrospective case series. J Chiropr Med. 2013;12(4):252-9. doi:10.1016/j.jcm. 2013.08.001.

29. McCrory P, Meeuwisse W, Johnston K, Dvorak J, Aubry M, Molloy M, et al. Consensus statement on Concussion in Sport-the 3rd International Conference on Concussion in Sport held in Zurich, November 2008. J Sci Med Sport. 2009;12(3):340-51. doi:10.1016/j.jsams.2009.02.004.

30. Guskiewicz KM, Register-Mihalik J, McCrory P, McCrea M, Johnston K, Makdissi $\mathrm{M}$, et al. Evidence-based approach to revising the SCAT2: introducing the SCAT3. Br J Sports Med. 2013;47(5):289-93. doi:10.1136/bjsports-2013-092225.

31. Bruce J, Echemendia R, Meeuwisse W, Comper P, Sisco A. 1 year test-retes reliability of ImPACT in professional ice hockey players. Clin Neuropsychol. 2014:28(1):14-25. doi:10.1080/13854046.2013.866272.

32. Lovell M, Maroon J, Collins M. The ImPACT Test. 2014. https://www. impacttest.com/about/?The-ImPACT-Test-4. Accessed March 312014.

33. Riemann B, Guskiewicz K, Shields EW. Relationship between clinical and forceplate measures of postural stability. J Sport Rehabil. 1999:8(2):71.

34. Finnoff JT, Peterson VJ, Hollman JH, Smith J. Intrarater and interrater reliability of the Balance Error Scoring System (BESS). PM R. 2009;1(1):50-4. doi:10.1016/j.pmrj.2008.06.002.

35. R Development Core Team. R: A language and environment for statistical computing. 3.0.0 ed. Vienna, Austria: R Foundation for Statistical Computing; 2013.

36. Wickham H. ggplot2: an implementation of the Grammar of Graphics. 0.9.3.1 ed. New York: Springer; 2013.

37. Kalanuria AA, Geocadin RG. Early prognostication in acute brain damage: where is the evidence? Curr Opin Crit Care. 2013;19(2):113-22. doi:10.1097/MCC.0b013e32835eba56.
38. Schulte S, Schiffer T, Sperlich B, Knicker A, Podlog LW, Struder HK. The impact of increased blood lactate on serum S100B and prolactin concentrations in male adult athletes. Eur J Appl Physiol. 2013:113(3):811-7. doi:10.1007/s00421-012-2503-9.

39. Kiechle K, Bazarian JJ, Merchant-Borna K, Stoecklein V, Rozen E, Blyth B, et al. Subject-specific increases in serum S-100B distinguish sports-related concussion from sports-related exertion. PLoS One. 2014;9(1):e84977. doi:10.1371/journal.pone.0084977.

\section{Submit your next manuscript to BioMed Central and we will help you at every step:}

- We accept pre-submission inquiries

- Our selector tool helps you to find the most relevant journal

- We provide round the clock customer support

- Convenient online submission

- Thorough peer review

- Inclusion in PubMed and all major indexing services

- Maximum visibility for your research

Submit your manuscript at www.biomedcentral.com/submit
C) Biomed Central 